\title{
Lorentz invariance and quantum gravity: an additional fine-tuning problem?
}

\author{
John Collins, ${ }^{1}$ Alejandro Perez, ${ }^{1}$ Daniel Sudarsky, ${ }^{1,2}$ Luis Urrutia, ${ }^{2}$ and Héctor Vucetich ${ }^{3,4}$ \\ ${ }^{1}$ Physics Department, Pennsylvania State University, University Park, PA 16802, USA \\ ${ }^{2}$ Instituto de Ciencias Nucleares, Universidad Nacional Autónoma de México, A. Postal 70-543, México D.F. 04510, México \\ ${ }^{3}$ Instituto de Física, Universidad Nacional Autónoma de México A. Postal 70-543, México D.F. 04510, México \\ ${ }^{4}$ Observatorio Astronómico, Universidad de La Plata, La Plata, Argentina
}

(Dated: 30 October 2004)

\begin{abstract}
Trying to combine standard quantum field theories with gravity leads to a breakdown of the usual structure of space-time at around the Planck length, $1.6 \times 10^{-35} \mathrm{~m}$, with possible violations of Lorentz invariance. Calculations of preferred-frame effects in quantum gravity have further motivated high precision searches for Lorentz violation. Here, we explain that combining known elementary particle interactions with a Planck-scale preferred frame gives rise to Lorentz violation at the percent level, some 20 orders of magnitude higher than earlier estimates, unless the bare parameters of the theory are unnaturally strongly fine-tuned. Therefore an important task is not just the improvement of the precision of searches for violations of Lorentz invariance, but also the search for theoretical mechanisms for automatically preserving Lorentz invariance.
\end{abstract}

The need for a theory of quantum gravity and a modified structure of space-time at (or before) the Planck scale is a consequence of the known and successful theories of classical general relativity (for gravity) and the standard model (for all other known interactions). Thus one of the most important challenges in theoretical physics is the construction of a quantum theory of gravitation.

Direct investigations of Planck-scale phenomena need short-wavelength probes with elementary-particle energies of order the Planck energy $E_{P}=\left(\hbar c^{5} / G\right)^{1 / 2}=$ $1.2 \times 10^{19} \mathrm{GeV}$, which is much too high to be practicable. But actual tests - e.g., 1, 2, 3] — of a hypothesized granularity of space-time at the Planck scale are possible because relativity (embodied mathematically as Lorentz invariance) gives a unique form for the dispersion relation between the energy and momentum of a particle,

$$
E=\sqrt{\mathbf{p}^{2} c^{2}+m^{2} c^{4}} .
$$

Here $c$, the speed of light is a universal constant, while the particle rest mass $m$ depends on the kind of particle. We will henceforth use units in which $c=1$.

Calculations in [4, 5] find preferred-frame effects associated with space-time granularity [ 6$]$ in the two most popular contenders for a theory of quantum gravity, which are string theory [9] and loop quantum gravity 10, 11]. In these scenarios, the preferred frame and the consequent Lorentz violation occur even though the fundamental classical equations of both of the theories are locally Lorentz invariant. We thus have a quantum inspired revival of the nineteenth century idea of the electromagnetic ether, a background in which propagate light waves, as well as all other elementary particles and fields. Specific estimates of modified dispersion relations were made in these papers from calculations of the propagation of quantum mechanical waves in the granular spacetime background. At accessible energies, only minute effects were predicted, of relative order $E / E_{P}$ or $\left(E / E_{P}\right)^{2}$, when the probe has energy $E$. For other ways in which

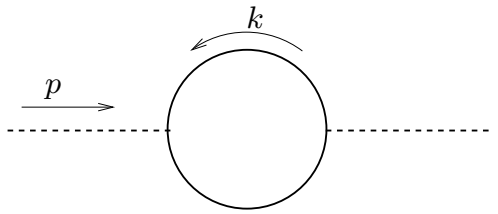

FIG. 1: Lowest order self-energy graph. Interactions of quantum fields require an unrestricted integral over the momenta of the virtual particles up to the highest momenta allowed in the theory.

Lorentz violation might arise, see, for example, 12, 13]. The minuteness of the effects is in accord with everyday scientific thinking, where we often find that the details of physical phenomena on one distance scale do not directly manifest themselves in physics on much larger scales. Therefore attention has focused on searches for extremely small violations of the dispersion relation.

However, as we will now explain, the predicted violations of the dispersion relations are enormously increased when we include known elementary particle interactions. In quantum field theories like the standard model, the propagation of an isolated particle has calculable contributions from Feynman diagrams for particle self-energies, such as Fig. 1. The dispersion law for a particle is obtained by solving

$$
E^{2}-\mathbf{p}^{2}-m^{2}-\Pi(E, \mathbf{p})=0 .
$$

Here $\Pi$ is the sum of all self-energy graphs, to which we have added any (small) Lorentz-violating corrections calculated in free-field theory as in [4, 5].

We now apply the following reasoning: Without a cutoff the graphs have divergences from large momenta/short distances. In the Lagrangian defining the theory, the divergences correspond to terms of dimension 4 (or less) that obey the symmetries of the microscopic theory. In the textbook situation with Lorentz invariance, the divergences are removed by renormaliza- 
tion of the parameters of the theory. Now Planck-scale physics can cut off the divergences, and also modify the formulae to be used when loop momenta are around the Planck scale. But the same power-counting that determines the divergences also determines the natural size of the contributions of Planck-scale virtual momenta: the dominant contributions correspond to operators of dimension 4 (or less) in the Lagrangian. (This is the argument that leads to the well-known concept of an effective low-energy theory.) If the microscopic theory violates Lorentz invariance at the Planck scale, then generically we also get Lorentz violation at low energies without any suppression by powers of $E / E_{P}$; for some details, see the Appendix. This Lorentz violation can be removed by explicit Lorentz-violating counterterms of dimension 4 in the Lagrangian which are fine-tuned to give the observed low-energy Lorentz invariance. But such fine-tuning is unacceptable 14, 15, 16 in a fundamental theory.

In the scenarios of [4, 5], Lorentz violating corrections to the dispersion relation, suppressed by one or two powers of $E / E_{P}$, were found by considering the propagation of free particles in a granular space-time background. The above reasoning shows, instead, that there are effects that are only suppressed by two powers of known standard-model couplings, effects that change the value of $c$ in the dispersion relation (11). The known, widely different, couplings of different elementary fields imply that different fields have different values of $c$, with fractional differences around $0.1 \%$ to $10 \%$. This is completely incompatible with the observed limits [1, 2, 3] that $c$ is constant at a fractional level well below $10^{-20}$.

This result is a direct consequence of well-known properties of quantum field theories, of which the standard model is only one example. Indeed the actual technical result is related to results that can be found more-orless explicitly in several of the cited references - e.g., by Kostelecký and Potting 17]. However, what appear to be almost uniformly missed or unemphasized are the consequences that we deduce. For example, Coleman and Glashow 1] and Colladay and Kostelecký [2, 18] recognize that the dominant effects of Lorentz violation at the Planck scale are represented by renormalizable [19] terms of dimension 4 or less in the standard model considered as a low-energy effective theory. Therefore they consider a Lorentz-violating extension of the standard model, and they set some of the observational bounds on the Lorentz violating parameters. However, we frequently see prominent statements, such as the one in [1], that Lorentz violation is "likely to be suppressed by some power of $\ldots m_{\mathrm{ew}} / M_{\mathrm{Pl}} \simeq 10^{-17}$," where $m_{\mathrm{ew}}$ is the electroweak scale. While this assumption is necessary to agree with experiment, it begs the question of whether the power suppression actually happens in a given theory with Planck-scale Lorentz violation.

Another example is the paper by Burgess et al. [12], who consider an effective field theory that arises from a higher dimensional scenario with Lorentz violation. At lowest order, Lorentz violation appears in the effective Lagrangian only in higher dimension graviton operators. Loop corrections involving gravitons have Lorentzviolating power-law divergences. These authors set the power-law divergences to zero, one justification for which is the use of dimensional regularization to define the effective theory. This appears to give only power-suppressed Lorentz violation, in contradiction with our estimates. However, one must also consider higher order contributions to the coefficients that relate the effective Lagrangian to the exact theory. Corresponding to the power-law divergences in the effective theory are finite contributions to coefficients, including those of Lorentzviolating operators of dimension 4 . The natural size of the Lorentz violation in their scenario is therefore the same as ours.

Finally, Myers and Pospelov [20] state an argument close to ours, but in the framework of effective field theory, with tree-approximation Lorentz violation given by higher dimension operators. They observe that loop corrections in the effective field theory give large Lorentz violation in accordance with our estimates. They then completely reverse their case by claiming to find a condition in which the Lorentz violation disappears at leading power. However, as explained by two of us [21], their argument does not work; even this second paper failed to take the next step, which is to realize that since a Planckscale Lorentz violation naturally leads to violations of observable Lorentz symmetry at the percent level, the considered scenarios are ruled out.

A closely related case, to which our arguments apply, is that of non-commutative field theories (NCFT), which may arise in a suitable limit from string theory [22]. In NCFT, a modified structure of space-time ("non-commutativity") appears as a Lorentz-violating non-locality in the action defining the field theory, with a characteristic scale $M$, which might be comparable with the Planck scale. Loop corrections in NCFT badly violate Lorentz invariance [23]. Our general argument applies to NCFT. Indeed, if NCFT is used without any extra UV cutoff, then loop momenta extend far above the non-commutativity scale in a highly Lorentzviolating manner. Thus these theories suffer from even more Lorentz violation than we have estimated. Indeed Anisimov et al. 13] have shown that NCFT (at least with a $c$-number non-commutativity tensor $\left.\theta_{\mu \nu}\right)$ are ruled out unless the UV cutoff $\Lambda$ is nine or ten orders of magnitude below the non-commutativity scale. Carlson, Carone and Lebed [24] have set even tighter limits in non-commutative QCD. Now the raison d'être of NCFT is to take account of non-standard space-time structure; but these already-known experimental limits show that NCFT cease to be applicable at scales far below the noncommutativity scale.

Notice that our argument has avoided explicit use of 
the language of effective field theory. Conventional treatments of effective field theory assume a Lorentz invariant cutoff for ultra-violet divergences; this cutoff is either dimensional regularization or a cutoff on momenta in $\mathrm{Eu}$ clidean space. In contrast, we examine the effect of a effective cutoff in Minkowski space, as provided by a putative granularity of real space-time.

The implications of our argument for both experiment and theory are quite profound. First, enthusiasm for improved searches for Lorentz violation should be dampened; given the stated motivations, the existing unsuccessful searches suffice by many orders of magnitude. Of course, it almost goes without saying that it is correct science to question and test accepted principles; on these grounds, tests of Lorentz invariance are worthwhile. But further enthusiasm has been engendered by estimates of specific small orders of magnitude for Lorentz violation. It is this excess enthusiasm that we wish to dampen.

As to theory, the critical task concerns any proposal in which Lorentz symmetry is substantially broken at the Planck scale. It is to find and implement a mechanism to give automatic local Lorentz invariance at low energies, despite a violation at the Planck scale. We assume here that the treatment involves real time, not an analytic continuation to imaginary time, as is common in treatments of quantum field theories in flat space-time. One mechanism is to have a custodial symmetry that is sufficient to prohibit Lorentz-violating dimension 4 terms, without itself being the full Lorentz group. But such a symmetry does not appear to be known. Corresponding issues arise in any proposal that involves modified dispersion relations, e.g., 25, 26, 27]; its proponents must show that the proposal survives experimental scrutiny after inclusion of known interactions.

Effectively we have shown that Lorentz invariance should be added to the well-known list of fine-tuning problems in the standard model; to date, this list is normally considered to include only the cosmological constant, the Higgs bare mass, and mass hierarchies.

There is not necessarily a conflict between discreteness and the absence of a preferred frame. In 28 Dowker et al. show how, by the use of a random causal set of points, space-time can be made discrete while Lorentz invariance is preserved in a suitable sense. From a more quantum-theoretic viewpoint, Rovelli and Speziale 29] argue that the existence of a minimum measurable length does not of itself imply that local Lorentz invariance is violated any more than the discreteness of the eigenvalues of the angular momentum operators implies violation of rotational invariance in ordinary quantum mechanics.

An optimistic point of view should be stressed: a branch of theoretical physics long considered to suffer from detachment from experimental guidance is now in the opposite situation. Because of mechanisms intimately tied to the known ultra-violet divergences in conventional quantum field theories, certain kinds of Planck- scale phenomena, like a preferred frame, manifest themselves suppressed by no powers of energy relative to the Planck energy, but only by two powers of standard model couplings. Lorentz invariance continues to play the powerful role it has played throughout the twentieth century of imposing stringent requirements on the kinds of mathematical theory that are permitted to agree with experiment.

Note added: After this paper was completed, a paper by Myers and Pospelov [30] appeared that contains a clear statement of the fine-tuning problem for Lorentz invariance. They deduce that the low-energy theory must have a cutoff far below the Planck scale, just as in our discussion of NCFT. However, they do not explain the problems of implementing such a cutoff Lorentz-invariantly in Minkowski space, as is appropriate in a theory of dynamical space-time.

Notice that the cutoff forms an upper limit to the validity of ordinary quantum field theory. If physics above the cutoff were represented by an ordinary quantum field theory, our argument would apply to that extended theory.

We would like to thank A. Ashtekar, J. Banavar, O. Bertolami, C. Burgess, Y. Chen, L. Freidel, L. Frankfurt, M. Graesser, A. Guijosa, J. Hall, T. Jacobson, J. Jain, C. Kozameh, A. Kostelecký, R. Montemayor, M. Mondragón, H.A. Morales-Técotl, H. Sahlmann, C. Stephens, and M. Strikman for useful discussions and comments. JC was supported in part by the U.S. Department of Energy. LFU acknowledges support from projects DGAPAIN11700 and CONACYT-40745-F. DS acknowledges support from the projects DGAPA-IN112401 and IN1081033 and CONACyT. AP acknowledges support from NSF grant PHY-0090091 and the Eberly Research Funds of Penn State University.

\section{ESTIMATE OF ONE-LOOP LORENTZ VIOLATION}

We now give some details of how Planck-scale Lorentz violation manifests itself in the propagation of low-energy particles, with a justification of our estimate of a percent for the observable effects. As a concrete example, we consider the self-energy graph of a scalar field with one fermion loop, Fig. 1 in a Yukawa theory. The main ideas are quite familiar and the general considerations involve only symmetry arguments and normal ultra-violet powercounting, which apply generally to all self-energy graphs.

Let $\Pi_{1}(p)$ represent the value of the graph. We quantify the dominant Lorentz violation at low energy by the quantity $\xi=\partial^{2} \Pi_{1}(p) / \partial\left(p^{0}\right)^{2}+\partial^{2} \Pi_{1}(p) / \partial\left(p^{1}\right)^{2}$ at $p=0$; this would be zero for a Lorentz-invariant self-energy.

Without the Planck-scale modifications, we would have

$$
\xi=-\frac{i g^{2}}{\pi^{4}} \int \mathrm{d}^{4} k \frac{\left[\left(k^{0}\right)^{2}+\left(k^{1}\right)^{2}\right]\left(k^{2}+3 M^{2}\right)}{\left(k^{2}-M^{2}+i \epsilon\right)^{4}} .
$$


Were it not that this integral is logarithmically divergent, it could be shown to be zero by continuing $k^{0}$ to imaginary values and then using Euclidean rotation invariance.

When there is Lorentz-violation in the free fermion propagator, as in [4, 5], the integrand would only apply in the low-energy limit. When $k$ is at or above the Planck scale, there would be substantial and Lorentz-violating modifications.

The would-be logarithmic divergence ensures that there is an order unity contribution from Planck-scale momenta, with their Lorentz violation. Note that a cutoff provided by preferred frame granularity is Lorentzviolating. As a simple illustration we modify the usual free-fermion propagator $i(\gamma \cdot k+m) /\left(k^{2}-m^{2}+i \epsilon\right)$ by a factor of a smooth function $f(|\mathbf{k}| / \Lambda)$ that obeys $f(0)=1$ and $f(\infty)=0$, with a cutoff parameter $\Lambda$. Then

$$
\xi=\frac{g^{2}}{6 \pi^{2}}\left[1+2 \int_{0}^{\infty} \mathrm{d} x x f^{\prime}(x)^{2}\right] .
$$

Thus the corresponding Lorentz violation is of order the square of the coupling independently of $\Lambda$. The exact value depends on the details of the Planck-scale free propagator, of course. The main point, however, is that the power counting that gives the would-be logarithmic divergence follows from standard arguments in the theory of renormalization, and that it applies to self-energy graphs for all fields. So the above integral gives a reasonable estimate of the size of the Lorentz violation, in the absence of some special cancellation. Typical measured standard-model couplings then give our percent estimate.

By use of (2), it can be checked that the $\xi$ coefficient corresponds to a modification of the dispersion relation (11) in which the speed of light parameter $c$ is modified by a factor $1+\xi / 4+O\left(\xi^{2}\right)$. In a theory with one field it is possible to treat this term as a renormalization of the space-time metric tensor which could remove the observable Lorentz violation. However, there are many fields in the standard model that differ by the sizes of their couplings. Hence renormalization of the metric tensor cannot remove all leading-power Lorentz violation.

Given a specific form of space-time granularity, accurate quantitative predictions could be obtained, with the use of renormalization-group methods etc. But such details would not affect the drastic disagreement of the percent estimate with actual observations.

[1] S.R. Coleman and S.L. Glashow, Phys. Rev. D 59, 116008 (1999).

[2] D. Colladay and V.A. Kostelecký, Phys. Rev. D 58, 116002 (1998).

[3] G. Amelino-Camelia, J.R. Ellis, N.E. Mavromatos, D.V. Nanopoulos, and S. Sarkar, Nature 393, 763 (1998). T. Jacobson, S. Liberati, and D. Mattingly, Nature 424,
1019 (2003). J. Alfaro and G. Palma, Phys. Rev. D67, 083003 (2003). R.J. Gleiser and C.N. Kozameh, Phys. Rev. D 64, 083007 (2001). D. Sudarsky, L. Urrutia, and H. Vucetich, Phys. Rev. Lett. 89, 231301 (2002). O. Bertolami, Gen. Rel. Grav. 34, 707 (2002).

[4] V.A. Kostelecký and S. Samuel, Phys. Rev. D 39, 683 (1989). G. Amelino-Camelia, J.R. Ellis, N.E. Mavromatos and D.V. Nanopoulos, Int. J. Mod. Phys. A 12, 607 (1997). J.R. Ellis, N.E. Mavromatos, and D.V. Nanopoulos, Phys. Rev. D 61, 027503 (2000). J.R. Ellis, K. Farakos, N.E. Mavromatos, V.A. Mitsou, and D.V. Nanopoulos, Astrophys. J. 535, 139 (2000). J. Alfaro, H.A. Morales-Tecotl, and L.F. Urrutia, Phys. Rev. D 66, 124006 (2002). H. Sahlmann and T. Thiemann, arXiv:gr-qc/0207031

[5] R. Gambini and J. Pullin, Phys. Rev. D 59, 124021 (1999).

[6] Note that Kozameh and Parisi [7] argue that the Lorentz violation found by Gambini and Pullin [5] in loop quantum gravity disappears when a change in canonical variables is made. But Alfaro et al. 8] argue that this change of variables is inconsistent with the quantization formalism used by Gambini and Pullin.

[7] C.N. Kozameh and M.F. Parisi, Class. Quant. Grav. 21, 2617 (2004).

[8] J. Alfaro, H.A. Morales-Tecotl, M. Reyes, and L.F. Urrutia, Phys. Rev. D 70, 084002 (2004).

[9] J. Polchinski, "String Theory," Vols. I and II, Cambridge University Press, 2000.

[10] C. Rovelli, Livings Reviews, 1, 1 (1998).

[11] T. Thiemann, Lect. Notes Phys. 631, 41 (2003).

[12] C.P. Burgess, J. Cline, E. Filotas, J. Matias, and G.D. Moore, J. High Energy Phys. 03 (2002) 043.

[13] A. Anisimov, T. Banks, M. Dine, and M. Graesser, Phys. Rev. D 65, 085032 (2002)

[14] S. Weinberg, Phys. Rev. D 8, 4482 (1973).

[15] L. Susskind, Phys. Rev. D 20, 2619 (1979).

[16] S. Weinberg, Rev. Mod. Phys. 61, 1 (1989).

[17] V.A. Kostelecký and R. Potting, Phys. Rev. D 51, 3923 (1995).

[18] D. Colladay and V.A.Kostelecký, Phys. Rev. D 55, 6760 (1997).

[19] V.A. Kostelecký, C.D. Lane, and A.G.M. Pickering, Phys. Rev. D 65, 056006 (2002).

[20] R.C. Myers and M. Pospelov, Phys. Rev. Lett. 90, 211601 (2003).

[21] A. Perez and D. Sudarsky, Phys. Rev. Lett. 91, 179101 (2003).

[22] N. Seiberg and E. Witten, J. High Energy Phys. 09 (1999) 032.

[23] S. Minwalla, M. Van Raamsdonk, and N. Seiberg, J. High Energy Phys. 02 (2000) 020.

[24] C.E. Carlson, C.D. Carone, and R.F. Lebed, Phys. Lett. B 518, 201 (2001)

[25] G. Amelino-Camelia, Int. J. Mod. Phys. D 11, 35 (2002).

[26] J. Magueijo and L. Smolin, Phys. Rev. Lett. 88, 190403 (2002).

[27] G. Amelino-Camelia, Nature 418, 34 (2002).

[28] F. Dowker, J. Henson, and R.D. Sorkin, Mod. Phys. Lett. A 19, 1829 (2004).

[29] C. Rovelli and S. Speziale, Phys. Rev. D 67, 064019 (2003)

[30] R.C. Myers and M. Pospelov, arXiv:gr-qc/0402028 